Auch bei der Interpretation der Isotopie-Effekte der Ionenwanderung in geschmolzenen Salzen ${ }^{13}$ (Reines $\mathrm{CdCl}_{2}: \mu_{+}=-0,067^{14}$, Pyrosol $\mathrm{CdCl}_{2}+\mathrm{Cd}$ : $\mu_{-}=-0,034^{12}$ ) war erkannt worden, daß beim Stromdurchgang gekoppelte Überführungen der elementaren Komponenten stattfinden, ohne daß die Art der Kopplung wirklich aufgeklärt werden konnte.

14 A. Klemm, H. Hintenberger u. W. Seelmann-Eggebert, Z. Naturforschg. 3 a, 172 [1948].
Es dürfte erhebliche Schwierigkeiten machen, hier zu präziseren Vorstellungen zu gelangen. Neue Informationen könnten sich aus Diffusions-Messungen und Messungen der äußeren Überführung an $\mathrm{CdCl}_{2}$ und $\mathrm{CdCl}_{2}+\mathrm{Cd}$-Pyrosol ergeben.

Herrn G. Josten danken wir für seine gewissenhafte Mitarbeit bei den Versuchen. Die Arbeit wurde durch Mittel des Bundesministeriums für Atomkernenergie und Wasserwirtschaft gefördert.

\title{
NOTIZEN
}

\section{Zum Cut-Off-Parameter der Impulsstreukoeffizienten von Systemen langreichweitiger Wechselwirkung}

Von G. Ecker und D. Voslamber

Institut für theoretische Physik der Universität Bonn (Z. Naturforschg. 15 a, 1107-1108 [1960]; eingeg. am 11. Oktober 1960)

Das dynamische Verhalten von Systemen mit langreichweitiger Wechselwirkung läßt sich an Hand der verallgemeinerten FоккеR-PLANK-Gleichung beschreiben. Diese Gleichung erfordert die Kenntnis der gemischten Impulsstreumomente

$$
\left\langle\left(\Delta p_{1}\right)^{\nu} \cdot\left(\Delta p_{2}\right)^{\left.\mu \cdot\left(\Delta p_{3}\right)^{\lambda}\right\rangle \begin{array}{l}
v+\mu+\lambda=n, \\
n=1,2, \ldots
\end{array},}\right.
$$

insbesondere der Momente erster und zweiter Ordnung. $\left(\Delta p_{1}, \Delta p_{2}, \Delta p_{3}\right)$ bedeutet die Impulsänderung des Testteilchens während eines geeignet gewählten Zeitintervalls $\Delta t$.

Die Impulsstreumomente sind bisher aus der Theorie des Mikrofeldes ${ }^{1,2}$ bzw. aus der stochastischen Überlagerung der Streubeiträge der einzelnen Feldteilchen unter der Voraussetzung vollständiger Passagen ${ }^{3-6}$ berechnet worden.

Während die Ergebnisse der ersten Methode von einer nur unscharf zu definierenden mittleren Lebensdauer des Mikrofeldes abhängen, treten bei der zweiten Methode logarithmisch divergierende Zahlwerte auf, die durch einen geeigneten Cut-Off-Parameter beschränkt werden müssen.

Das Auftreten der Divergenzen wird durch die beiden folgenden Näherungen verursacht:

1 S. Chandrasekhar, Astrophys. J. 94, 511 [1941].

2 S. Chandrasekhar, Astrophys. J. 97, 255, 263 [1943].

3 R. S. Cohen, L. Spitzer u. P. McRoutly, Phys. Rev. 80, 230 [1950].

4 S. Chandrasekhar, Principles of Stellar Dynamics, Univ. Chicago Press 1942.

5 D. Bонм u. L. H. Aller, Astrophys. J. 105, 131 [1947].

${ }^{6}$ L. Spitzer, Physics of Fully Ionized Gases, Intersci. Tracts on Physics and Astronomy, New York 1956.
1. Die Vernachlässigung der Korrelation der Feldteilchen untereinander. Diesem Effekt läßt sich in erster Näherung durch Berücksichtigung der Debyeschen $\mathrm{Ab}$ schirmung in der Wahl des Cut-Off-Parameters ${ }^{3}$ bzw. in der Theorie des Mikrofeldes ${ }^{7}$ Rechnung tragen. Für ein Zweikomponentenensemble resultiert hieraus bereits die Beseitigung der Divergenzschwierigkeiten. Jedoch gilt das gleiche nicht für ein Einkomponentenensemble.

2. Die Voraussetzung vollständiger Teilchenpassagen ist eine Näherung, die insbesondere für entferntere Feldteilchen nicht gerechtfertigt ist. Die Berücksichtigung dieser Tatsache bringt die Divergenzschwierigkeiten auch für den Fall des Einteilchenensembles ohne Abschirmung zum Verschwinden.

Um dies zu erkennen, ist es notwendig, die einzelnen Streuanteile der Feldteilchen während eines Zeitintervalls $\Delta t$ mit Hilfe der bekannten, stochastischen Methoden $\mathrm{zu}$ überlagern ${ }^{8}$. Die Rechnung wird allerdings durch die endliche Länge der Bahnen der Feldteilchen erheblich kompliziert ${ }^{9,10}$. Sie wirkt sich nur auf das auch in den Cut-Off-Theorien auftretende Logarithmusglied aus, welches wir hier mit $L$ bezeichnen wollen. Dieses ist, wenn der Debye-Radius als begrenzender Parameter benutzt wird,

$$
L_{\mathrm{h}}=\log \left[\frac{(k T)^{3 / 2} M}{(\varkappa \pi)^{1 / 2} \gamma e^{3} m n^{1 / 2}}\right] .
$$

Unsere Berücksichtigung der Endlichkeit der Flugbahnen ${ }^{9}$ liefert für ein Einkomponentenensemble von Teilchen der Ladung $e$

$$
L_{\mathrm{I}}=\log \left[\frac{2 \Delta t M}{e^{3} \gamma^{3 / 2}}\left(\frac{2 k T}{m}\right)^{3 / 2}\right],
$$

7 G. Ecker u. K. G. Müller, Z. Phys. 148, 593 [1957].

8 S. Chandrasekhar, Rev. Mod. Phys. 15, 1 [1943].

9 G. ECKer u. D. Voslamber, Forschungsbericht d. L. Nrdh. Westf. Nr. 832 (1959); ähnliche Ergebnisse wurden auch von M. HeNoN ${ }^{10}$ erzielt, jedoch mit astro-physikalischer Blickrichtung. Diese Arbeit ist uns erst nach Abschluß unserer Untersuchungen bekannt geworden (Phys. Abstr. Mai 1960).

10 M. Henon, Ann. d'Astrophysique 21, 186 [1958]. 
für ein Zweikomponentenensemble (Plasma) dagegen

$$
L_{\mathrm{II}}=L_{\mathrm{I}}-L_{\mathrm{m}}\left[2 \Delta t e\left(\frac{\pi n}{\varkappa m}\right)^{1 / 2}\right],
$$

wo

$$
\begin{aligned}
L_{\mathrm{m}}[s]=\int_{0}^{\infty}\left[K_{0}^{2}(x)+K_{1}^{2}(x)\right] x & \\
& \cdot\left[\Phi(s x)-\begin{array}{c}
1 \\
s x \sqrt{\pi}
\end{array}\right.
\end{aligned}
$$

eine Funktion ist, deren maschinelle Auswertung vorliegt ${ }^{9}$. Es gelten die Bezeichnungen: $K_{0}, K_{1}$ modifizierte Hankel-Funktion; $\Phi$ Gausssches Fehlerintegral; $m$ Masse der Feldteilchen; $M$ reduzierte Masse vom Test- und Feldteilchen; $n$ Trägerdichte; $e$ Elementarladung; $T$ Temperatur; $k$ Boltzmannsche Konstante; $\log \gamma=0,577$ Eulersche Konstante; $x$ ist gleich 1 oder 2 zu setzen, je nachdem es sich um Elektronen oder Protonen handelt.

Wir bemerken, daß $L_{\mathrm{I}}$ und $L_{\mathrm{II}}$ noch von $\Delta t$ abhängen. Die Streubeiträge konsekutiver Zeitintervalle können daher nicht mehr völlig stochastisch unabhängig sein. Dieses zunächst überraschende Ergebnis ist konsequent, da das Testteilchen im Laufe zweier aufeinanderfolgender Zeitintervalle von entfernteren Feldteilchen im gleichen Sinne beeinflußt wird. Im Hinblick auf die Anwendung in der FоKKER-PLANK-Gleichung haben wir diese Korrelation so klein wie möglich zu halten, d. h. wir müssen das Zeitintervall $\Delta t$ so groß wie möglich wählen. Andererseits müssen wir aus Gründen der Modellvorstellung $\Delta t$ kleiner wählen als das Zeitintervall $\tau$, während dem die Feld- und Testteilchen merklich aus der ursprünglichen Richtung abgelenkt werden. Folglich haben wir in den Gln. (3) und (4) $\Delta t$ mit $\tau$ identifiziert.

Die Abb. 1 demonstriert neben unseren Resultaten $L_{\mathrm{I}}$ und $L_{\mathrm{II}}$ die entsprechenden Größen der früheren Rechnungen mit Cut-Off beim mittleren Teilchenabstand $\left(L_{0}\right)$ bzw. beim Debyeschen Radius $\left(L_{\mathrm{h}}\right)$.

Ersichtlich stimmen die neuen Resultate für das Zweikomponentenensemble $\left(L_{\mathrm{II}}\right)$ im Bereiche $T \cdot n^{-1 / s}>10$ mit den Ergebnissen des Cut-Off beim Debye-Radius überein. Dagegen zeigen sich im Bereich $T \cdot n^{-1 / 3}<10$ Abweichungen, die davon herrühren, da $\beta$ selbst innerhalb der Debye-Zone um das Testteilchen die Flugbahnen der Feldteilchen während $\Delta t$ nicht mehr als unendlich lang angesehen werden dürfen. Allerdings wird in diesem Gebiet mit abnehmendem $T \cdot n^{-1 / 3}$ das Ergebnis aller Rechnungen wegen Versagens der Modellvorstellung allmählich mit Unsicherheiten behaftet.

Für das Einkomponentenensemble ergibt sich fast im gesamten Bereich der Abb. 1 ein erheblicher Unterschied zwischen $L_{\mathrm{I}}$ und $L_{0}$. Für hohe Temperaturen und geringe Dichten gilt $L_{\mathrm{I}}: L_{\mathrm{II}}: L_{0}=3: 2: 1$. Geeignete, gute Beispiele für homogene Einkomponentenensemble

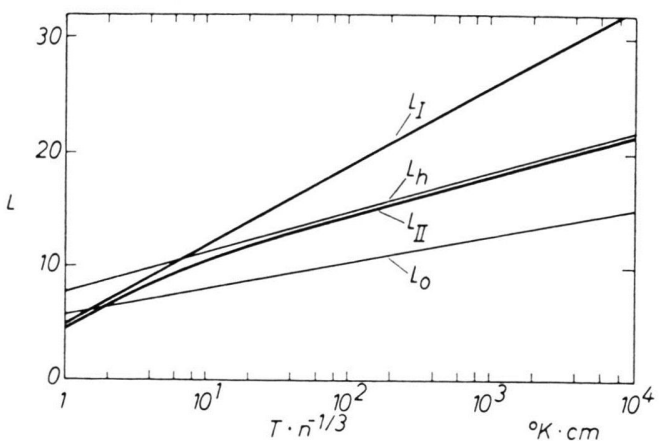

Abb. 1.

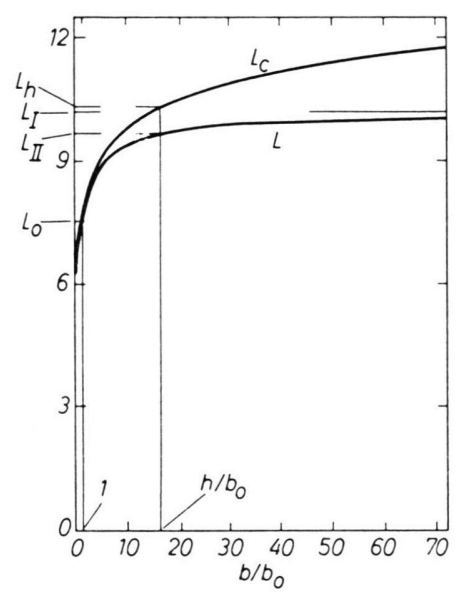

Abb. 2.

kommen in der Natur nur sehr selten vor. Raumladungszonen zeigen allgemein gewisse Inhomogenitäten. Bei Sterngesamtheiten ist - wie Henon ${ }^{10}$ gezeigt hat infolge Clusterbildung ebenfalls der Ausdruck $L_{\mathrm{h}}$ und nicht $L_{\mathrm{I}}$ zu verwenden.

Um schließlich die Beiträge der einzelnen Bereiche der Umgebung des Tetsteilchens beurteilen zu können, haben wir in der Abb. 2 für $T \cdot n^{-1 / 3}=5,5 L$ als Funktion eines maximalen Stoßparameters $b$ aufgetragen. $b_{0}$ ist der mittlere Teilchenabstand. Das Ergebnis der CutOff-Theorien ist durch die Kurve $L_{\mathrm{c}}$ gekennzeichnet, das neue Ergebnis durch $L$. Während die Funktion $L_{\mathrm{c}}$ mit wachsendem, maximalem Stoßparameter logarithmisch über alle Grenzen wächst, nähert sich $L$ mit wachsendem $b / b_{0}$ dem Grenzwert $L_{\mathrm{I}}$, der in der Figur als Asymptote eingezeichnet ist. Die den verschiedenen Cut-Off-Parametern (mittlerer Teilchenabstand bzw. Deвye-Länge) zugeordneten Zahlwerte $L_{0}, L_{\mathrm{h}}, L_{\mathrm{II}}$ sind in der Figur ebenfalls vermerkt. 